VIII ${ }^{\text {èmes }}$ Journées Nationales Génie Civil - Génie Côtier, Compiègne, 7-9 septembre 2004

\title{
Evolution morphodynamique des souilles d'extraction marines
}

\author{
P. Gomi ${ }^{(a)}$, P. Sergent ${ }^{(a)}$, B. Zhang ${ }^{(b)}$ \\ (a) Centre d'Etudes Techniques Maritimes et Fluviales 2, Boulevard Gambetta, 60200 Compiègne, tel: \\ 03.44.92.60.30,patrick.gomi@equipement.gouv.fr \\ (b) Université de Technologie de Compiègne; 60, Avenue de Landshut, 60200 Compiègne, tel: \\ 03.44.23.46.99.(0).41.49, bainian.zhang@utc.fr
}

\section{Résumé}

Les fosses d'extraction de granulats marins sont une source intéressante de matériaux et peuvent se substituer à l'extraction classique en carrière notamment lorsqu'il y a un besoin de matériaux de remblais pour des infrastructures portuaires ou off-shore mais également pour recharger les plages et les dunes en érosion. Néanmoins il est important d'envisager l'influence que peut avoir une fosse d'extraction sur le transport sédimentaire de la région où elle se trouve ainsi que d'étudier à quelle vitesse cette fosse d'extraction piège le sédiment et se remplit de nouveau. Dans le cadre du projet européen Sandpit, plusieurs outils de modélisation numérique sont comparés sur des cas-tests schématiques puis validés sur des sites d'études réels.

\section{Abstract}

Submarine mining pits are interesting material sources and can replace standard quarries especially when there are needs of filling materials for building harbour facilities or off-shore structures and also for eroded beach or dune filling. However it is important to be able to estimate correctly the impact of submarine mining pits on the local sediment transport and on their filling rate. One task of the European project Sandpit is to develop and compare several sand transport modelling tools on simple physical test cases and then to validate these models on real test cases.

\section{Introduction}

Le recours à des fosses d'extraction marines de granulats en eaux peu profondes (de 10 à 30 mètres de profondeurs) va, à l'avenir, être de plus en plus fréquent dans de nombreux pays européens. Ainsi en Mer du Nord et en Méditerranée il va falloir trouver de nouvelles sources de sable afin de recharger les plages et les dunes côtières soumises à une érosion amplifiée par l'élévation attendue du niveau des mers. De plus, de nombreuses régions côtières ont besoin d'emprises de constructions à grande échelle (infrastructures portuaires et aéroportuaires) qui vont nécessiter des quantités considérables de matériaux de remblais. Pour toutes ces raisons les fosses d'extraction marines existantes vont être considérablement étendues et de nouveaux sites potentiels vont être prospectés. Ces fosses d'extraction en mer ont bien sûr un impact significatif sur les paramètres hydrodynamiques locaux mais également sur des régions plus éloignées pouvant aller jusqu'à la côte. Une fosse d'extraction marine a tendance naturellement à se combler et à se comporter comme un puits à sédiments compensé par une érosion sédimentaire des fonds aux alentours de la souille. Un nouveau site d'extraction en mer doit être situé pas trop loin des côtes pour des coûts d'exploitation acceptables mais pas non plus trop près du littoral pour éviter d'ajouter une source d'érosion supplémentaire dans la région côtière. 
Le recours à la modélisation numérique dans le cadre du projet européen Sandpit va permettre de répondre à de nombreuses questions comme connaître les dimensions et la géométrie optimales d'une fosse pour minimiser les coûts et ses effets sur l'érosion côtière, déterminer la position des sites à privilégier, estimer le temps de remplissage d'une souille une fois celle-ci épuisée, appréhender l'effet d'une exploitation massive sur le potentiel en sédiments de la région. Dans cette étude, nous présenterons les résultats de nos modèles numériques, déjà utilisés pour étudier l'évolution d'un dépôt de clapage ${ }^{1}$, sur les deux cas tests schématiques du projet Sandpit ainsi que les résultats obtenus sur le site hollandais de Scheveningen. Les données et les étapes préalables à la modélisation de la souille Cnexo sont aussi présentées à la fin de cet article.

\section{Modèles utilisés}

\subsection{Modèle d'évolution des fonds}

La conservation de la quantité de sédiment sur tout le domaine d'étude se traduit par l'équation classique d'évolution des fonds:

$$
(1-p) \frac{\partial z_{f}}{\partial t}+\operatorname{div}\left(q_{s}\right)=0
$$

où $p$ est la porosité des fonds, $z_{f}$ la cote des fonds, $q_{s}$ le débit solide total qui peut s'écrire comme la somme du débit solide de sédiments charriés et du débit solide de sédiments en suspension soit $q_{s}=q_{s c}+q_{s s}$. Plusieurs lois de transport expriment ce débit de transport total en fonction des différents paramètres physiques du problème et pour tous les exemples traités par le Laboratoire d'Hydraulique Numérique (LHN) dans le cadre du projet européen Sandpit et développés dans la suite de cet article nous avons utilisé les lois de Bijker ${ }^{2}$ et la loi de Soulsby-Van Rijn ${ }^{3}$. Dans le cas où le transport en suspension est modélisé en utilisant une équation de convection-diffusion, l'équation d'évolution des fonds se met alors sous la forme suivante où $S$ est un terme source défini dans le paragraphe 2.2:

$$
(1-p) \frac{\partial z_{f}}{\partial t}+\operatorname{div}(q s c)=-S
$$

\subsection{Modèle de transport des sédiments en suspension}

Lorsque le transport sédimentaire par suspension est conséquent il est important de coupler le modèle d'évolution des fonds avec un module de type convection-diffusion régi par l'équation suivante:

$$
\frac{\partial c}{\partial t}+u \frac{\partial c}{\partial x}+v \frac{\partial c}{\partial y}=\frac{\partial}{\partial x}\left(D_{x x} \frac{\partial c}{\partial x}\right)+\frac{\partial}{\partial y}\left(D_{y y} \frac{\partial c}{\partial y}\right)+\frac{1}{h} S
$$

- $u$ et $v$ sont les composantes horizontales de la vitesse moyennées sur la verticale ;

- $c$ est la concentration des sédiments en suspension moyennée sur la verticale ;

- $D_{x x}$ et $D_{y y}$ sont les coefficients de diffusion du sédiment en suspension ;

- $S$ est le terme source permettant de modéliser à partir de quel moment le sédiment se met en suspension ou au contraire se redépose.

On considère pour notre modèle que la diffusion du sédiment est homogène et isotrope suivant les deux directions horizontales et on choisit $D_{x x}=D_{y y}=1 \mathrm{~m}^{2} \mathrm{~s}^{-1}$ pour tous les calculs. Le terme source s'exprime généralement de la façon suivante : $S=\alpha W_{S}\left(c_{S^{-}}-c\right)$ avec $W_{s}$ la vitesse de chute du sédiment tandis que $c_{S}$ est la concentration de sédiment à l'équilibre moyennée 
sur la verticale donnée par Van Rijn ${ }^{4}$. Pour tous nos calculs nous avons choisi $\alpha$ égal à 1 comme recommandé par de nombreux auteurs.

\subsection{Modèle de houle et de courantologie}

Le modèle d'évolution des fonds utilisé actualise automatiquement les vitesses du courant par conservation du débit. Il suffit juste alors de calculer le champ de courants initial en utilisant le modèle aux éléments finis Reflux résolvant les équations de St-Venant et qui donne pour chaque nœud du maillage les deux composantes horizontales de la vitesse moyennées sur la verticale ainsi que le niveau d'eau. Pour certains tests il a fallu modéliser la déformation du champ de houle due à la présence de la souille car de forts gradients de bathymétrie peuvent engendrer de forts gradients de hauteurs de houle et, comme on retrouve les vitesses orbitales de la houle dans les lois de transport total, cela peut conduire à de fortes variations par rapport à un champ de houle que l'on considérerait homogène. Ce calcul d'agitation se fait en utilisant le modèle aux éléments finis Refonde résolvant l'équation de Berkhoff pour des ondes monochromatiques ou aléatoires. Il est également possible et envisageable de coupler les deux modèles décrits ci-dessus pour obtenir les courants de houles dus aux contraintes de radiation de celles-ci. Néanmoins dans tous les tests décrits par la suite, ces courants de houle sont négligeables par rapport au courant moyen.

\section{Cas tests schématiques}

\subsection{Test 1: houle perpendiculaire à l'axe de la tranchée}

Ce cas test physique a été mené par Delft Hydraulics ${ }^{5}$ dans un bassin pouvant générer houles et courants. Le fond est constitué de sables fins $\left(d_{50}=100 \mu \mathrm{m}, d_{90}=130 \mu \mathrm{m}\right)$ et une houle irrégulière est produite par un générateur directionnel suivant un spectre de Jonswap ayant une fréquence de pic de $0,4 \mathrm{~Hz}$. La hauteur d'eau est de $0,4 \mathrm{~m}$ pour tous les tests. En tout, il y a eu 27 conditions de test avec trois hauteurs de houle, trois directions de houle et trois valeurs de courants différentes. Le cas étudié pour le projet Sandpit est une unique configuration de ce cas test physique avec une houle de hauteur significative $\mathrm{H}_{\mathrm{s}}$ égale à $0,1 \mathrm{~m}$, de période de pic $T_{\mathrm{p}}$ égale à 2,16 $\mathrm{s}$ et perpendiculaire à un courant valant environ $0,2 \mathrm{~ms}^{-1}$. Trois profils de cette tranchée ont été relevés régulièrement pendant 25 heures. Les dimensions initiales de cette tranchée sont une profondeur d'environ 0,2 $\mathrm{m}$, une largeur au fond d'environ $0,5 \mathrm{~m}$ et des pentes d'environ 1 pour 8 (voir figure 1). Ce cas test a été spécialement conçu pour mettre en valeur une action de la houle prédominante sur le transport sédimentaire et un calcul d'agitation avec la bathymétrie initiale montre que le champ de houle est très déformé par la présence de la souille et qu'il est préférable d'actualiser ce champ de houle au cours de la modélisation où le fond évolue assez rapidement. Le transport sédimentaire se fait ici essentiellement par charriage et il est inutile de coupler notre module d'évolution des fonds avec le module de convection-diffusion qui réclame un temps de calcul beaucoup plus important. La condition aux limites en sortie est ouverte alors que nous fixons un $z$ constant en entrée pour modéliser l'apport constant en sédiments.

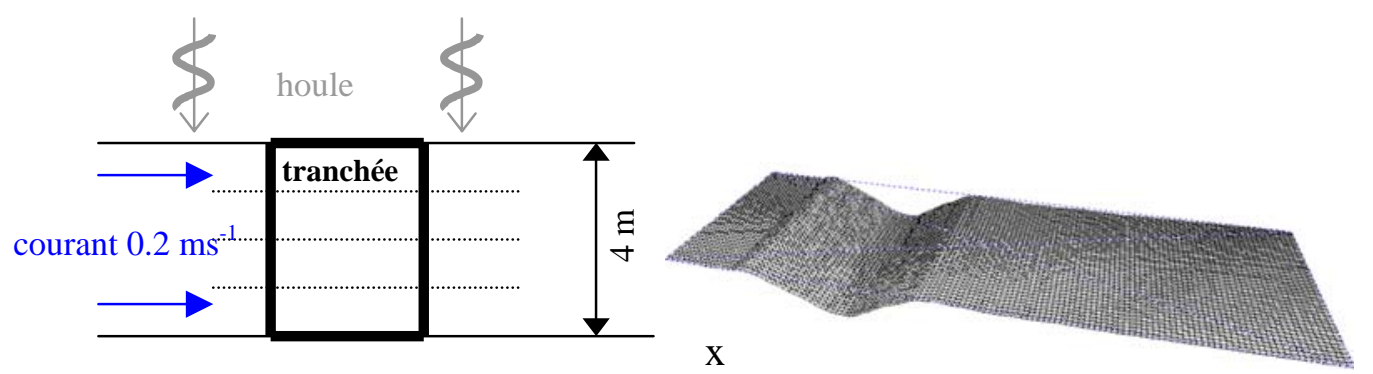


Figure 1: Conditions expérimentales pour le Test 1

Une première modélisation de cette fosse en négligeant la houle et en ne prenant en compte que le courant ne déclenche aucun mouvement sédimentaire ce qui montre l'importance de bien prendre en compte la houle pour ce test. Un calcul en utilisant un champ de houle constant et homogène sur tout le domaine donne curieusement des résultats plutôt bons alors que si on lance le calcul avec un champ de houle constant dans le temps mais calculé avec Refonde en utilisant la bathymétrie initiale nous obtenons des résultats très moyens avec notamment la formation d'une bosse importante en amont et qui s'accélère avec le temps. C'est la raison pour laquelle nous avons décidé d'actualiser le champ de houle toutes les 30 minutes en prenant en compte la modification de la bathymétrie ce qui donne des résultats légèrement meilleurs que pour une houle constante. Cependant, pour toutes ces configurations, on se rend compte que le fond de la fosse se comble difficilement et surtout que la pente amont de cette fosse se raidit trop par rapport à ce qui se passe en réalité. Cette constatation a été faite par tous les modélisateurs du projet Sandpit qui ont entrepris la modélisation de ce test 1 . C'est pourquoi nous avons décidé de modifier légèrement la loi de Soulsby-Van Rijn (SVR) en multipliant l'influence de la houle dans la loi de transport total par un coefficient $\alpha_{S V R}$ (à l'origine égal à 1) dont le profil tend à diminuer le transport en haut de la fosse (pour éviter de raidissement des pentes) mais également à augmenter le transport solide au fond de la fosse pour la faire se remplir plus rapidement et de façon moins anguleuse. Cette modification est d'autant plus légitime que les conditions de ce test (profondeur d'eau, vitesse moyenne, diamètre moyen) se trouvent aux limites des conditions d'applications de la loi. Tous les résultats décrits ci-dessus sont représentés sur la figure 3 tandis que la loi modifiée de Soulsby-Van Rijn est écrite ci-dessous avec le coefficient $\alpha_{S V R}(h)$ décrit sur la figure 2 ( $\mathrm{K}_{\mathrm{V}}$ étant un paramètre dépendant de la porosité des dépôts).

$$
Q_{S}=K_{V} \cdot A_{S} \cdot \bar{U} \cdot\left[\sqrt{\bar{U}^{2}+\alpha_{S V R}(h) \cdot \frac{0.018}{C_{D}} U_{r m s}^{2}}-\bar{U}_{c r}\right]^{2.4}
$$

\section{h}
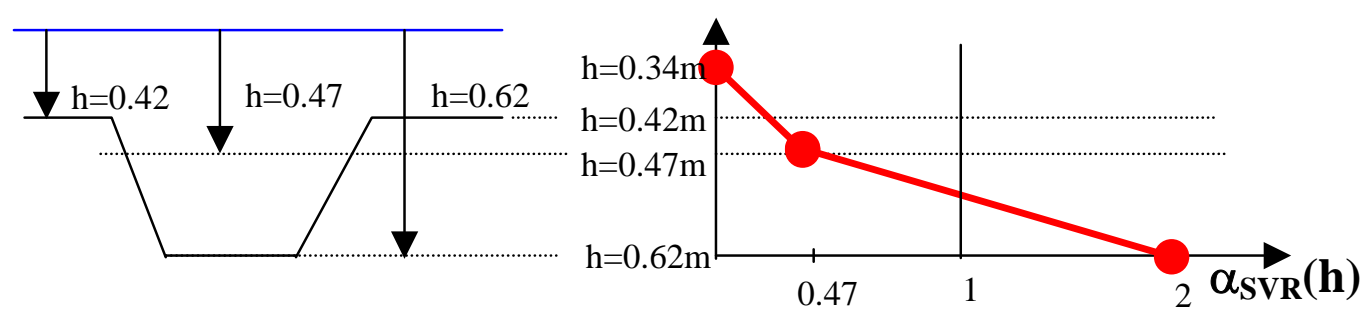

Figure 2: Représentation du paramètre $\alpha(h)$ modifiant l'équation de SVR.

La calibration de notre modèle s'est effectué en modifiant uniquement le coefficient de Strickler. Le coefficient de Strickler calculé en utilisant les paramètres physiques du test de Delft Hydraulics donne un coefficient $K_{s}$ égal à 88 qui ne donne lieu à aucun mouvement sédimentaire dans notre modèle numérique. Nous avons tout d'abord effectué une calibration 
légère en prenant un coefficient de Strickler de 70 qui donne des résultats satisfaisants en ce qui concerne la bathymétrie malgré un transport sédimentaire nul au fond de la fosse et qui engendre un raidissement de la pente amont de cette fosse.

Le transport solide initial est aussi sous évalué par rapport à ce qui a été observé pendant l'expérience physique où le débit solide

en entrée était d'environ $0,02 \mathrm{~m}^{3} / \mathrm{s}$ (voir figure 4). Pour la loi de Soulsby-Van Rijn modifiée nous avons effectué une calibration plus forte du Strickler ( $K_{s}$ égal à 42$)$ et la loi de SoulsbyVan Rijn non-modifiée donne alors un taux de transport initial proche de la réalité. Choisir des coefficients 70 et 42 à la place de 88 prévu par les données du test permet de diminuer la vitesse critique respectivement d'un facteur $1,25(=88 / 70)$ et $2,09(=88 / 42)$ ce qui permet d'obtenir un mouvement sédimentaire au fond de la fosse et donc un remplissage.

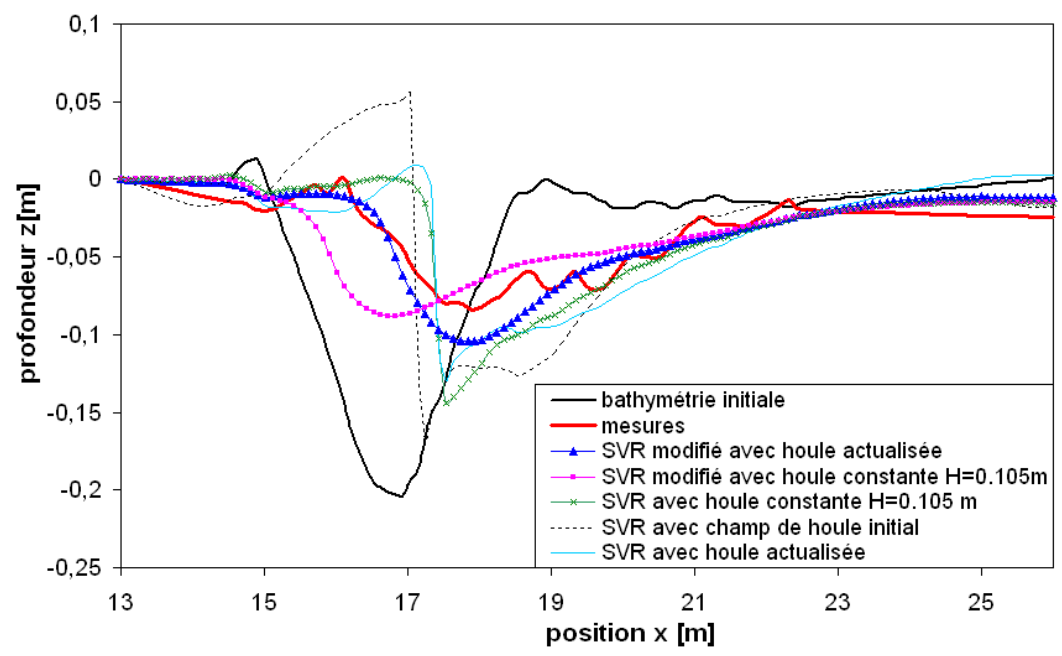

Figure 3: Profils finaux obtenus pour le Test 1 suivant plusieurs lois de transport.

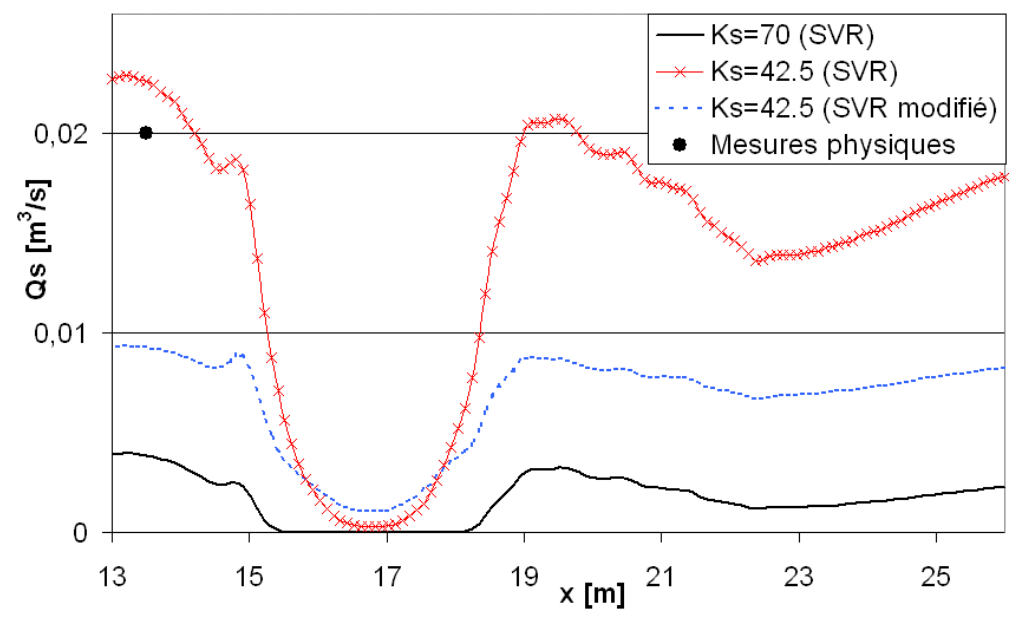

Figure 4: Taux de transport initial pour le test 1 pour plusieurs calibrations. 


\subsection{Test 2: houle parallèle à l'axe de la tranchée}

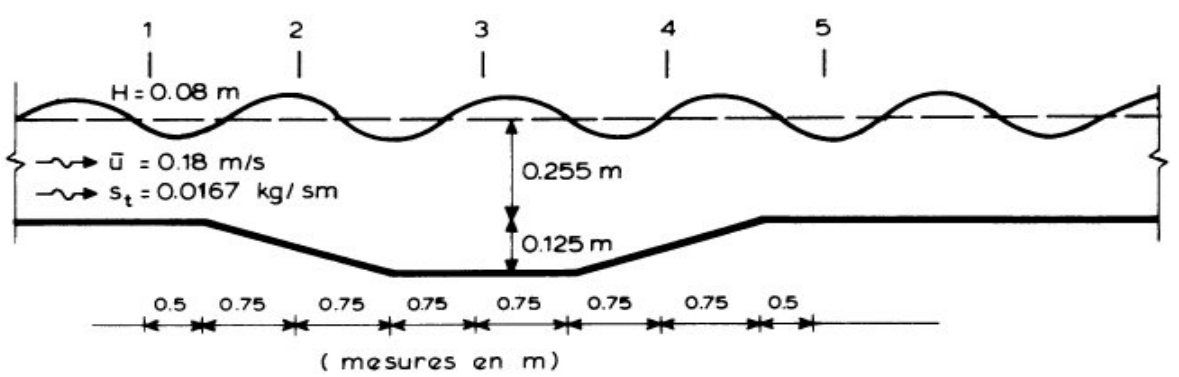

Figure 5: Conditions expérimentales pour le test 2.

Ce cas test 2 s'appuie également sur des essais physiques réalisés par Delft Hydraulics ${ }^{6}$ dans un canal à houle de $17 \mathrm{~m}$ de long, de $0,3 \mathrm{~m}$ de large et de $0,5 \mathrm{~m}$ de profondeur. Le sable utilisé a un diamètre moyen $d_{50}$ de $0,1 \mathrm{~mm}$ et $d_{90}$ de $0,13 \mathrm{~mm}$. En entrée du canal soumis à un courant constant de vitesse $u=0,18 \mathrm{~ms}^{-1}$ on introduit un débit constant de sédiment calculé pour compenser exactement la quantité de sédiment qui sort du domaine. La fosse elle même a des pentes de 1 pour 12 et une profondeur de $0,125 \mathrm{~m}$. La durée du test est de 10 heures. La houle régulière se propage dans le sens du courant et a une hauteur de $8 \mathrm{~cm}$ avec une période de 1,5 s (voir figure 5). Dans ce cas test, l'influence de la houle sur le transport sédimentaire n'est pas aussi sensible que dans le premier cas et nous utilisons un champ de houle calculé avec Refonde au temps initial. Nous calculons également le champ de courant initial avec Reflux qui sera lui actualisé automatiquement par conservation du débit.

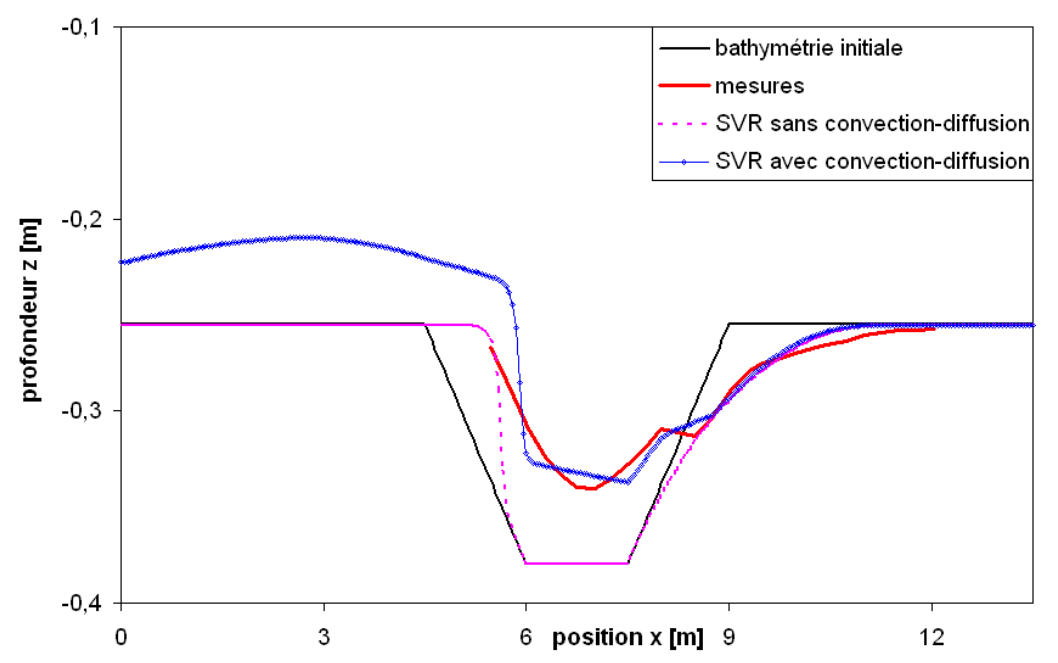


Figure 6: Profils finaux obtenus pour le test 2 avec ou sans convection-diffusion.

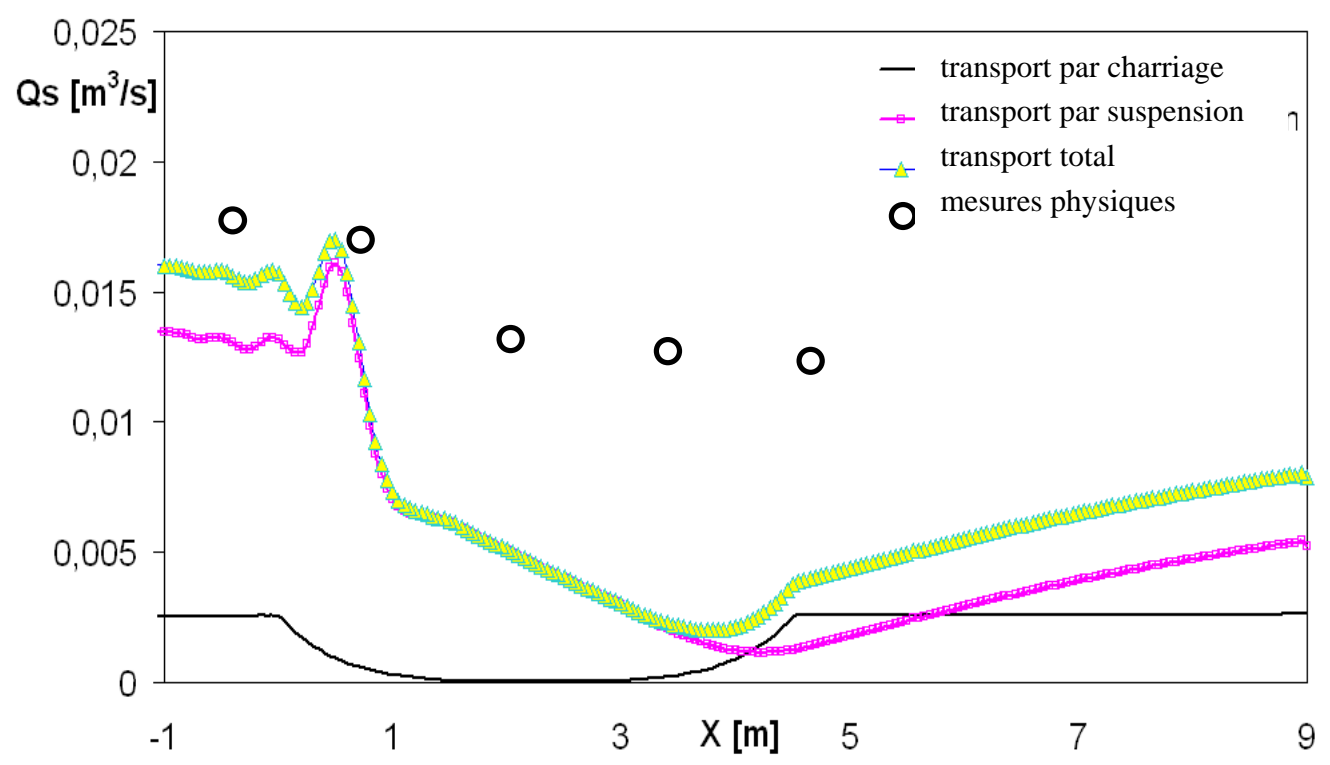

Figure 7: Taux de transport initial pour le test 2.

Ce test a été spécialement conçu pour étudier le transport sédimentaire par suspension comme le montrent les figures 6 et 7 et cela quel que soit le coefficient de Strickler utilisé. C'est pourquoi nous utilisons la loi de Soulsby-Van Rijn pour modéliser uniquement le transport par charriage alors que le transport par suspension est modélisé par le modèle de convection-diffusion décrit ci-dessus. La condition aux limites en sortie est ouverte alors que nous imposons un débit solide constant de sédiments en suspension en entrée. Le transport de sédiment sur tout le domaine d'étude ne semble pas à l'équilibre et notre modèle crée une zone de dépôt importante en amont de la fosse qui n’apparaît pas avec le modèle physique. Les résultats obtenus avec notre modèle couplé sont plutôt satisfaisants hormis le dépôt de sédiments excessif en amont de la fosse. Le remplissage de cette dernière est correct même si on rencontre les mêmes problèmes de raidissement de la pente amont qui est due à une absence de transport par charriage au fond de la fosse. On peut noter également que taux de transport initial est fidèle aux mesures en amont car on l'utilise comme conditions aux limites mais qu’il est sous évalué par notre modèle pour le reste du domaine.

\section{Cas test de Scheveningen}

Une fosse expérimentale a été creusée en Mer du Nord près de la ville hollandaise de Scheveningen en Mars 1964 dans le but d'obtenir des informations sur les vitesses de remplissage de cette fosse, de creuser et d'enfouir un pipeline. Cette tranchée expérimentale a été creusée perpendiculairement à la cote à une distance variant entre $1 \mathrm{~km}$ (profondeur d'eau valant alors $7 \mathrm{~m}$ ) et 1,7 km (profondeur d'eau valant alors 10,5 m). Cette tranchée a une longueur de $700 \mathrm{~m}$, sa largeur au fond est d'environ $10 \mathrm{~m}$ tandis que ses pentes sont à peu près de 1 pour 10 . La profondeur de cette fosse est d'environ $2 \mathrm{~m}$ en dessous du niveau 
moyen des fonds dans lesquels elle est creusée. En tout $30.000 \mathrm{~m}^{3}$ ont été extraits. Le sable est relativement fin avec un diamètre moyen $D_{50}$ variant entre 0,2 et $0,25 \mathrm{~mm}$ et un diamètre $D_{90}$ variant entre 0,4 et $0,5 \mathrm{~mm}$. Cette tranchée à la géométrie régulière a été instrumentée pendant 175 jours (du 7 Mars 1964 au 7 Août 1964) montrant un remplissage symétrique de cette dernière mais aucun déplacement.

Contrairement aux deux cas tests physiques présentés précédemment, la fosse est soumise à des marées qui génèrent des courants perpendiculaires à cette dernière (entre $+0,6 \mathrm{~ms}^{-1}$ et $0,5 \mathrm{~ms}^{-1}$ ) et un niveau d'eau variant suivant une courbe périodique simplifiée de période 12 heures dont l'amplitude varie entre $+1.5 \mathrm{~m}$ et $-1 \mathrm{~m}$. La modélisation numérique de cette fosse expérimentale a été en fait simplifiée et ramenée à une étude similaire au test 2 en considérant néanmoins un niveau d'eau variable soumis à une marée régulière et un histogramme de houle simplifié. Les conditions aux limites sont posées de la même façon que pour le test 1 . Deux lois de transport de Bijker et de Soulsby-Van Rijn ont été utilisées et ont donné lieu à des comportements légèrement différents. La houle a ici un effet important sur le transport sédimentaire et des houles très faibles génèrent peu ou pas du tout de transport. Le climat de houle proposé pour cette étude comporte quelques jours de tempêtes où la houle devient conséquente $\left(\mathrm{H}_{\mathrm{p}}=3,7 \mathrm{~m}\right.$ et $\left.\mathrm{T}_{\mathrm{p}}=8,1 \mathrm{~s}\right)$ pendant lesquels les mouvements sédimentaires sont importants. Une modélisation avec la loi SVR en utilisant des données de houle brutes mène à un remplissage quasi-total de la fosse et nous avons légèrement modifié le climat de houle en prenant en compte l'interaction houle-courant. En effet une houle qui se propage en eau peu profonde et qui rencontre un courant fort voit ses caractéristiques modifiées par ce dernier. Nous avons utilisé les lois proposées par Van Rijn pour modifier les propriétés de la houle ( $\mathrm{T}$, $\mathrm{H}_{\mathrm{p}}$ et $\mathrm{L}_{\mathrm{p}}$ ) ce qui conduit à une diminution sensible de la hauteur de houle et en contre-partie à un remplissage moindre de la souille qui reste néanmoins surestimé par rapport aux mesures réelles. L'utilisation de la loi de Bijker sans modifier le climat de houle donne lieu au contraire à une sous-estimation du remplissage de la souille malgré le choix $b=5$ dans cette loi ( $b=2$ étant généralement recommandé) et un coefficient de Chezy valant 55. Les résultats obtenus pour ces deux lois sont regroupés sur la figure 8.

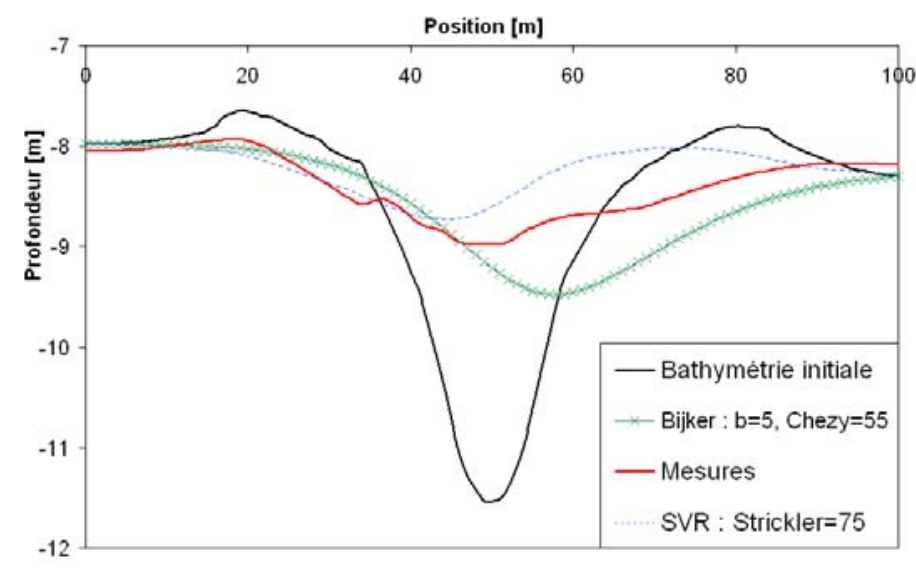

Figure 8: Profils finaux obtenus pour le test 3 pour les lois de Bijker et de SVR.

\section{$\underline{\text { 5.Cas test de la souille Cnexo }}$}


Le creusement de la fosse expérimentale du Cnexo a commencé en 1973 afin d'étudier en grandeur nature les impacts que peuvent engendrer les exploitations sous-marines de sédiments. Plusieurs campagnes bathymétriques et granulométriques ont été menées sur ce site dans le but d'étudier plus précisément la faculté de cette souille à se remplir ainsi que ses répercussions sur le transport sédimentaire dans les régions aux alentours.

Comme un grand jeu de données est disponible sur ce site, les modélisateurs du programme Européen Sandpit ont décidé de le retenir comme cas test réel de validation des modèles développés et testés au préalable sur les trois cas-tests présentés auparavant. Une base de données complète a été collectée à l'usage de ces modélisateurs.

\subsection{Présentation générale de la souille Cnexo}

La souille Cnexo se situe au large de l'estuaire de la Seine, est longue d'environ 2,5 km et a une largeur moyenne d'environ $400 \mathrm{~m}$. Elle est orientée SO-NE et a été creusée dans une région où les fonds varient entre $16 \mathrm{~m}$ et $17,5 \mathrm{~m}$ avec un léger dénivelé vers le Nord. Ses coordonnées géographiques sont : Latitude comprise entre $49^{\circ} 30^{\prime} \mathrm{N}$ et $49^{\circ} 31^{\prime} \mathrm{N}$ et Longitude comprise entre $0^{\circ} 06^{\prime} 15^{\prime}$ ' $\mathrm{W}$ et $0^{\circ} 08^{\prime} 30^{\prime}$ ' $\mathrm{W}$. Les extractions de la souille Cnexo ont eu lieu de 1974 à 1980 à travers 13 campagnes qui ont enlevé plus de $2.800 .000 \mathrm{~m}^{3}$ de sédiments. Néanmoins tout le domaine concédé n’a pas été exploité en même temps et on distingue deux régions ${ }^{7}$ :

(a) de 1974 à 1977, seule la partie Nord-Est du domaine a été exploitée sur une longueur d'environ $1500 \mathrm{~m}$. A la fin de cette première étape, cette "vieille » partie de la souille est large de $200 \mathrm{~m}$ et a une profondeur variant entre 3 et $5 \mathrm{~m}$.

(b) de 1977 à 1980, la partie Sud-Ouest du domaine est aussi creusée plus profondément et plus étroitement. A la fin de la dernière campagne d'extraction en 1980, la fosse en son entier a une longueur d'environ $3 \mathrm{~km}$ et une largeur variant entre 130 et $300 \mathrm{~m}$. Elle est plus profonde dans sa partie Sud-Ouest (entre $5 \mathrm{~m}$ et $13 \mathrm{~m}$ ) que dans sa partie Nord-Est (entre $3 \mathrm{~m}$ et $6 \mathrm{~m})$.

\subsection{Evolution bathymétrique de la souille Cnexo}

Trois campagnes bathymétriques ont été effectuées en 1981, 1996 et 2002. Les deux bathymétries les plus récentes ont été relevées par le Port du Havre qui a utilisé exactement le même système de positionnement GPS dans les deux cas. Le Port du Havre considère qu'avec cette méthode la précision est d'environ $1 \mathrm{~m}$ pour les coordonnées planimétriques et inférieure à $20 \mathrm{~cm}$ pour les coordonnées altimétriques. La bathymétrie de 1981 est moins fiable que les précédentes et a été obtenue en digitalisant une carte marine de cette époque. Même si cette campagne bathymétrique de 1981 a été menée avec soins, on ne peut pas s'attendre à avoir une précision meilleure que 10 à $15 \mathrm{~m}$ pour les coordonnées planimétriques et $30 \mathrm{~cm}$ pour les coordonnées altimétriques. Armés de ces trois relevés bathymétriques, nous avons analysé l'évolution volumique sur tout le domaine, l'évolution volumique de la souille seule pour cibler son comportement propre, l'évolution de plusieurs profils en travers et le long de la souille et l'évolution des cartes bathymétriques pour localiser plus précisément les régions en érosion et en accrétion (voir figure 9). Toutes ces analyses permettent de constater un remplissage important de la souille entre 1981 et 1996 tandis que les deux pentes la constituant sont érodées, ce qui semble physiquement correct. Le même genre d'évolution est plus difficilement discernable entre 1996 et 2002. Les mouvements sédimentaires les plus conséquents ont lieu dans la partie Sud de la souille qui est la plus étroite et la plus creusée. 

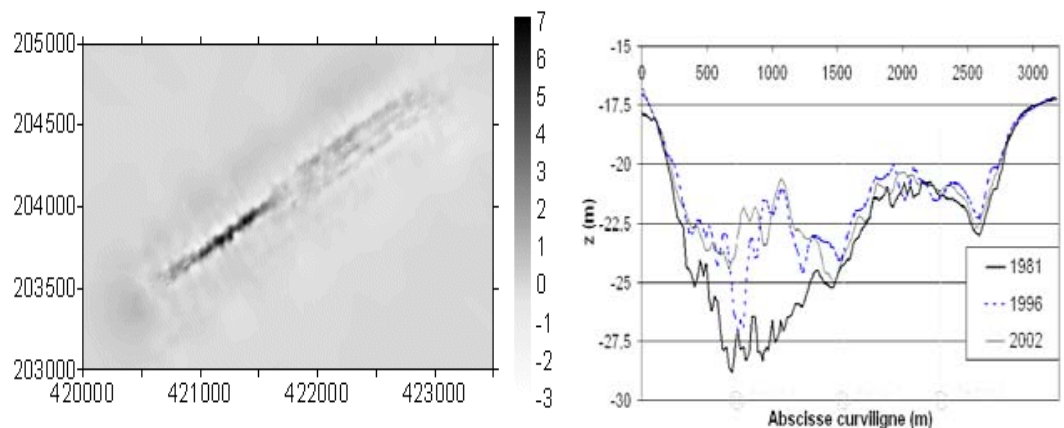

Figure 9: Carte bathymétrique différentielle entre 1996 et 1981 (à gauche) et évolution du profil en long (à droite).

\subsection{Données de houle et déformation de la houle au dessus de la souille}

Des données de houle ont également été collectées en utilisant deux bouées situées aux alentours de la souille et qui appartiennent au réseau français d'observation des vagues CANDHIS. Cette base de données est très complète mais malheureusement les directions de la houle n'ont pas encore été extraites ce qui est pénalisant. En effet une étude d'agitation de la houle menée avec le logiciel aux éléments finis Refonde (équation de Berkhoff) montre que la direction de houle est un paramètre très important dans ce cas présent. L'atlas Numérique de houle des côtes françaises développé dans un projet commun entre Météo-France, CETMEF et EDF-LNHE a permis d'extraire pour les années 1999 et 2000 les propriétés de la houle $\left(\mathrm{H}_{\mathrm{p}}, \mathrm{T}_{\mathrm{p}}\right.$, Direction) toutes les trois heures, soient 5800 mesures. Une analyse complète de cette base de données numériques a été entreprise pour isoler les directions principales de la houle et créer des histogrammes de houle plus ou moins sophistiqués à l'usage des modélisateurs du projet Européen Sandpit.

\subsection{Données hydrodynamiques et granulométriques}

La souille Cnexo se trouve sous influence macrotidale ce qui peut entraîner de grandes disparités dans les champs hydrodynamiques de vitesses. Les coefficients théoriques de marées ont été collectés depuis la base de données du SHOM. Sogreah a également proposé d'extraire les données concernant la souille Cnexo de leur modèle numérique de l'estuaire de la Seine. Les résultats extraits sont donnés entre le $1^{\mathrm{er}}$ Avril 2002 et le 30 Avril 2002 ce qui correspond à 56 cycles de marées avec un coefficient minimal de 33 (6 Avril 2002) et un coefficient maximal de 112 (27 Avril 2002). Ces résultats couplés avec la base de données du SHOM permettent d'interpoler n'importe quel autre coefficient de marée et donc de trouver n'importe quel champ hydrodynamique entre 1981 et 2002.

Desprez $^{8}$ du Groupe d’Etudes des Milieux Estuariens et Littoraux a fourni des données granulométriques pour quatre échantillons à l'intérieur de la souille et six échantillons dans son voisinage proche Les caractéristiques moyennes du sédiment extrait sont résumées dans le tableau 1. 
VIII ${ }^{\text {èmes }}$ Journées Nationales Génie Civil - Génie Côtier, Compiègne, 7-9 septembre 2004

Tableau 1: Caractéristiques moyennes des échantillons granulométriques à l'intérieur de la souille et dans son voisinage.

\begin{tabular}{|c|c|c|}
\hline & Intérieur souille & Voisinage \\
\hline \% graviers $(>2 \mathrm{~mm})$ & 9.4 & 1.4 \\
\hline \% sables grossiers $(0.5-2 \mathrm{~mm})$ & 14.3 & 11.9 \\
\hline \% sables moyens $(\mathbf{2 0 0}-\mathbf{5 0 0} \boldsymbol{\mu m})$ & $\mathbf{5 4 . 1}$ & $\mathbf{6 8 . 9}$ \\
\hline \% sables fins $(50-200 \mu \mathrm{m})$ & 21.6 & 16.4 \\
\hline \% particules fines $(<50 \mu \mathrm{m})$ & 2.9 & 0.6 \\
\hline Diamètre moyen $(\mu \mathrm{m})$ & 393 & 388 \\
\hline
\end{tabular}

\section{Conclusion}

Une meilleure compréhension des influences que peuvent avoir les fosses d'extraction de granulats marins sur l'environnement proche est nécessaire pour mieux appréhender leur dimensionnement et leur localisation. Un des objectifs du projet européen Sandpit est dédié à cette problématique en utilisant comme outil la modélisation numérique du transport sédimentaire. Deux cas-cas tests schématiques (test 1 et test 2) ont été soumis aux modèles numériques de nombreux organismes européens dont le LHN ainsi qu'un cas-test réel simplifié (test 3). Ces trois cas-tests préliminaires sont complémentaires et permettent à chaque fois d'isoler un phénomène physique prédominant (influence de la houle dans le test 1 , influence du transport par suspension dans le test 2, niveau d'eau variable dans le test 3). Ils ont permis de montrer la pertinence d'utiliser les modèles numériques pour étudier l'évolution des fosses numériques que ce soit à court terme ou à moyen terme. Ces études mettent également en valeur les limites actuelles des lois de transport classiques qui doivent être parfois modifiées pour mieux représenter certaines sensibilités physiques. Le modèle développé et calibré sur ces trois premiers cas-tests sera par la suite validé sur le site réel du Cnexo pour lequel une base de données exhaustive a été établie à l'usage des modélisateurs du projet Sandpit.

\section{Références}

1.Sergent, P., Zhang, B., 2002, Evolution du dépôt de clapage du Kannick, VII ièmes journées nationales Génie Civil Génie Côtier, Anglet, pp 195-204

2.Bijker, E.W., 1968, Mechanics of sediment transport by the combination of waves and current in design and reliability of coastal structures, $23^{\text {rd }}$ Int. Conf. On Cosatal Engineering, pp 147-173

3.Soulsby R., 1997, Dynamics of marine sands, Thomas Thelford Edition

4.Van Rijn L.C., 1984, Sediment transport Part I: Bed load transport, Journal of Hydraulic Engineering, ASCE, 110 (10), pp1431-1456 Part II: Suspended load transport, Journal of Hydraulic Engineering, ASCE, 110 (11), pp1613-1641

5.Walstra, D.J.R., Van Rijn, L.C., Hoogewoning, S.E et Aarninkhof, S.G.J, 1999, Modeling of sedimentation of dredged trenches and channels under the combined actions of tidal currents and waves. Coastal sediments 1999.

6.Van Rijn L.C., 1986, Sedimentation of dredged channels by currents and waves, Journal of Waterway, Port, Coastal and Ocean Engineering, ASCE, 112 (5), pp 541-559

7.Lemoine M., Clabaut P., Simon S., Augris C., 1999, Étude de la souille expérimentale d'exploitation de granulats marins dite "souille CNEXO » en baie de Seine : évolution morpho-sédimentologique et faunistique entre 1981 et 1996, Rapport Ifremer. 
8.Desprez M., 1996, Étude des sédiments superficiels et de la macrofaune benthique dans le secteur de l'ancienne fouille expérimentale du CNEXO. État en décembre 1995. Rapport GEMEL Picardie. 